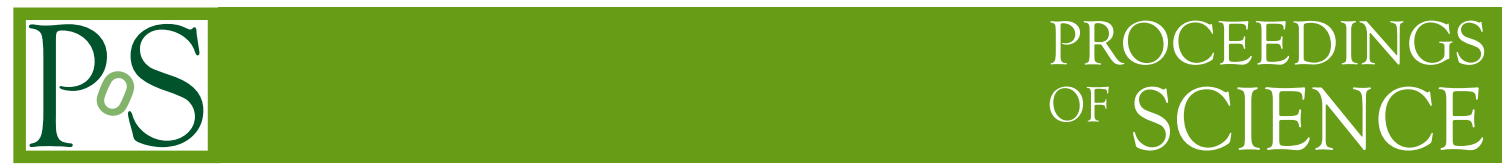

\title{
Radiative production of the hidden-charm pentaquarks
}

\section{E. Ortiz-Pacheco ${ }^{a, *}$ and R. Bijker ${ }^{a}$}

${ }^{a}$ Instituto de Ciencias Nucleares, Universidad Nacional Autónoma de México, 04510, Ciudad de México, México

E-mail: emmanuelo@ciencias.unam.mx, bijker@nucleares.unam.mx

In the present work we estimate the orbital contribution to the photoproduction of the ground state pentaquarks, described as compact $q q q Q \bar{Q}$ configurations with $J^{P}=3 / 2^{-}$in the framework of the harmonic oscillator quark model. The photocouplings $P_{c}(u u d c \bar{c}) \rightarrow p+\gamma$ are found to be highly suppressed due to the large value of the photon momentum.

*** 10th International Workshop on Charm Physics (CHARM2020), ***

***31 May - 4 June, 2021 ***

*** Mexico City, Mexico - Online ***

${ }^{*}$ Speaker 


\section{Introduction}

In 2015, for the first time. LHCb observed two signals in the $J / \psi p$ channel of the $\Lambda_{b}^{0} \rightarrow$ $J / \psi p K^{-}$decay, consistent with a charmonium-pentaquark state with minimal quark content $u u d c \bar{c}$ $[1,2]$. The measured masses of these resonances were $4380 \pm 8 \pm 29 \mathrm{MeV}$ and $4449.8 \pm 1.7 \pm$ $2.5 \mathrm{MeV}$, and the pentaquarks were denoted as $P_{c}(4380)$ and $P_{c}(4450)$, respectively. The preferred assignments $J^{P}$ were suggested with opposite parity, and with one state having spin $3 / 2$, while the other 5/2. More recently, in 2019 with more data and statistics the LHCb collaboration discovered a new narrow pentaquark state $P_{c}(4312)^{+}$with a mass of $4311.9 \pm 0.7_{-0.6}^{+6.8} \mathrm{MeV}$ [3]. In addition, it was found that the pentaquark signal $P_{c}(4450)^{+}$consists of two narrow peaks $P_{c}(4440)^{+}$with a mass of $4440.3 \pm 1.3_{-4.7}^{+4.1} \mathrm{MeV}$ and $P_{c}(4457)^{+}$with $4457.3 \pm 0.6_{-1.7}^{+4.1} \mathrm{MeV}$. Currently, there are still many interpretations trying to provide an explanation for all these detected signals [4-10].

Apart from many theoretical studies to interpret and comprehend the nature of the pentaquark signals, there are experimental efforts to try to confirm them in other production channels. One of the ways to confirm the nature of the pentaquark signal is to photoproduce the states $P_{c}$ using an electromagnetic probe: $p(u u d)+\gamma \rightarrow P_{c}(u u d c \bar{c})$. The GlueX experiment located in Hall D at Jefferson Lab has had, as one of its main purposes, the search and confirmation of the announced pentaquark states by $\mathrm{LHCb}$, through electron scattering experiments based on $J / \psi$ photoproduction [11-15]. In 2019, The GlueX Collaboration published the first results of the collected data, where in the conclusions of their analysis they did not see evidence, to date, for the pentaquark signals by this mechanism [16]. Since in none of those experiments the quantum numbers of these resonances were determined, their understanding and interpretation remains an open problem.

In this contribution, we treat the pentaquark states as compact five-quark configurations, as for example in Refs. [17-22]. In a previous study of pentaquark states in the constituent quark model we discussed a complete classification of the ground state pentaquarks [22]. However, no distinction was made between light and heavy quarks. The aim of this contribution is to present the first results of a harmonic oscillator quark model analysis of pentaquarks in which we do distinguish between light and heavy quarks and, moreover, make an explicit calculation of the photocouplings which are relevant for photoproduction experiments.

\section{Pentaquark wave function}

In order to construct the pentaquark wave functions for ground states, we implement two conditions: for the first one, the pentaquark wave function should be antisymmetric under any permutation of the three light quarks, and for the second one, as all physical states, it should be a color singlet. The permutation symmetry is labeled by $A_{1}, A_{2}$ and $E$ for the symmetric, antisymmetric and mixed symmetry representations, respectively.

In this contribution, we discuss the ground-state pentaquark configurations with $J^{P}=3 / 2^{-}$. Since the orbital part of pentaquark wave function with $L^{P}=0^{+}$is symmetric $\left(A_{1}\right)$, the color-spinflavor part has to be antisymmetric $\left(A_{2}\right)$

$$
\psi=\left[\psi_{A_{1}}^{\mathrm{o}} \times \psi_{A_{2}}^{\mathrm{csf}}\right]_{A_{2}},
$$




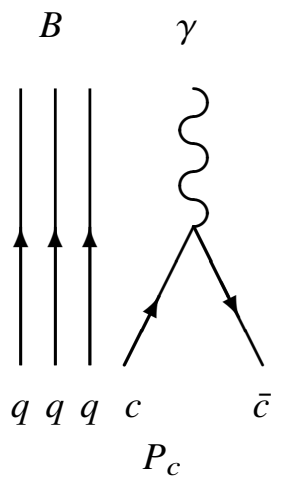

Figure 1: Electromagnetic decay of pentaquark $P_{c}$ into a baryon $B$ and a photon, $P_{c} \rightarrow B+\gamma$.

Due to the symmetry properties of the color part, the spin-flavor part is either symmetric $\left(A_{1}\right)$ or mixed symmetric $(E)$ under the permutation of the three light quarks

$$
\begin{aligned}
& \psi_{A_{2}}^{\mathrm{csf}}=\left[\psi_{A_{2}}^{\mathrm{c}} \times \psi_{A_{1}}^{\mathrm{sf}}\right]_{A_{2}}, \\
& \psi_{A_{2}}^{\mathrm{csf}}=\left[\psi_{E}^{\mathrm{c}} \times \psi_{E}^{\mathrm{sf}}\right]_{A_{2}} .
\end{aligned}
$$

\section{Photocouplings of hidden-charm pentaquarks}

In this section we study the photocouplings for ground state pentaquarks. The Hamiltonian for electromagnetic couplings is given by [23]

$$
H=e \int d^{3} x \hat{J}^{\mu}(\vec{x}) A_{\mu}(\vec{x})
$$

where $A_{\mu}$ is the electromagnetic field and $J^{\mu}$ is the quark current

$$
\hat{J}^{\mu}(\vec{x})=\sum_{q} \bar{q}(\vec{x}) e_{q} \gamma^{\mu} q(\vec{x}) .
$$

The prime interest of this work is calculate the photocouplings for pentaquarks, in particular an electromagnetic decay process between the pentaquark (configuration $q q q c \bar{c}$ ) and the proton $(q q q)$, hence the relevant term for this decay process $P_{c} \rightarrow p+\gamma$ is the annihilation of a $c \bar{c}$ pair, see Fig. 1. The three light quarks $q q q$ are spectators and the photo-coupling is to the quark-antiquark pair $c \bar{c}$.

The radiative decay widths for pentaquark states can be calculated from the helicity amplitudes as [24-26]

$$
\Gamma\left(P_{c} \rightarrow B+\gamma\right)=2 \pi \rho \frac{2}{2 J+1} \sum_{v>0}\left|A_{v}(k)\right|^{2},
$$

where $\rho$ is the phase-space factor and $k$ is the photon momentum. The helicity amplitude for this process is defined as the transition matrix element between the pentaquark and proton

$$
\begin{aligned}
A_{\nu}(k) & =\left\langle B, 1 / 2, v-1 ; \gamma|H| P_{c}, 3 / 2, v\right\rangle \\
& =\frac{e}{(2 \pi)^{\frac{3}{2}} 2 \sqrt{k_{0}}}\left\langle\psi_{B}^{c s f}, \frac{1}{2}, v-1\left|e_{c} \sigma_{-}\right| \psi_{P_{c}}^{c s f}, \frac{3}{2}, v\right\rangle F(k) .
\end{aligned}
$$


The helicity amplitude factorizes into a product of a color-spin-flavor matrix element and a form factor, $F(k)$, representing the orbital matrix element. The color-spin-flavor matrix part gives a non-vanishing contribution only for the first pentaquark wave function of Eq. (2) with

$$
\psi=\frac{1}{\sqrt{2}} \psi_{A_{1}}^{o} \psi_{A_{2}}^{c}\left(\phi_{E_{\rho}} \chi_{E_{\rho}}+\phi_{E_{\lambda}} \chi_{E_{\lambda}}\right)
$$

\section{Harmonic oscillator quark model}

The form factor, $F(k)$, can be evaluated analytically in the harmonic oscillator quark model

$$
H=\frac{p_{1}^{2}+p_{2}^{2}+p_{3}^{2}}{2 m}+\frac{p_{4}^{2}+p_{5}^{2}}{2 m^{\prime}}+\frac{1}{2} C \sum_{i<j}^{5}\left(\vec{r}_{i}-\vec{r}_{j}\right)^{2},
$$

with one mass for the light quarks, $m=m_{u / d}$, and another for the heavy quarks, $m^{\prime}=m_{c}$. It is convenient to make a change of variables to the center-of-mass coordinate, $\vec{R}$, the relative Jacobi coordinates for the light quarks, $\vec{\rho}$ and $\vec{\lambda}$, and the heavy quarks, $\vec{\eta}$, and a relative coordinate between the light and heavy quarks, $\vec{\zeta}$,

$$
\begin{aligned}
\vec{\rho} & =\frac{1}{\sqrt{2}}\left(\overrightarrow{r_{1}}-\overrightarrow{r_{2}}\right) \\
\vec{\lambda} & =\frac{1}{\sqrt{6}}\left(\overrightarrow{r_{1}}+\overrightarrow{r_{2}}-2 \overrightarrow{r_{3}}\right) \\
\vec{\eta} & =\frac{1}{\sqrt{2}}\left(\overrightarrow{r_{4}}-\overrightarrow{r_{5}}\right) \\
\vec{\zeta} & =\sqrt{\frac{6}{5}}\left[\frac{1}{3}\left(\vec{r}_{1}+\overrightarrow{r_{2}}+\overrightarrow{r_{3}}\right)-\frac{1}{2}\left(\overrightarrow{r_{4}}+\overrightarrow{r_{5}}\right)\right] \\
\vec{R} & =\frac{m\left(\overrightarrow{r_{1}}+\overrightarrow{r_{2}}+\overrightarrow{r_{3}}\right)+m^{\prime}\left(\vec{r}_{4}+\vec{r}_{5}\right)}{3 m+2 m^{\prime}} .
\end{aligned}
$$

In the new coordinates the Hamiltonian separates into the center-of-mass motion and four independent harmonic oscillators in $\rho, \lambda, \eta$ and $\zeta$

$$
H=\frac{P^{2}}{2 M}+\frac{p_{\rho}^{2}}{2 m_{\rho}}+\frac{p_{\lambda}^{2}}{2 m_{\lambda}}+\frac{p_{\eta}^{2}}{2 m_{\eta}}+\frac{p_{\zeta}^{2}}{2 m_{\zeta}}+\frac{5}{2} C\left(\rho^{2}+\lambda^{2}+\eta^{2}+\zeta^{2}\right),
$$

with

$$
M=3 m+2 m^{\prime}, \quad m_{\rho}=m_{\lambda}=m, \quad m_{\eta}=m^{\prime}, \quad m_{\zeta}=\frac{5 m m^{\prime}}{3 m+2 m^{\prime}} .
$$

The corresponding harmonic oscillator constants and frequencies are given by

$$
\alpha_{\mu}^{2}=\sqrt{5 C m_{\mu}}, \quad \omega_{\mu}=\sqrt{5 C / \mu}, \quad \mu=\{\rho, \lambda, \eta, \zeta\} .
$$

The oscillator constant for the pentaquark is related to the charge radius by

$$
\left\langle r_{\mathrm{ch}}^{2}\right\rangle_{P_{c}}=\frac{1}{\alpha_{\rho}^{2}}\left(1+\frac{1}{5}\left(\frac{5 a}{3+2 a}\right)^{\frac{3}{2}}\right) .
$$



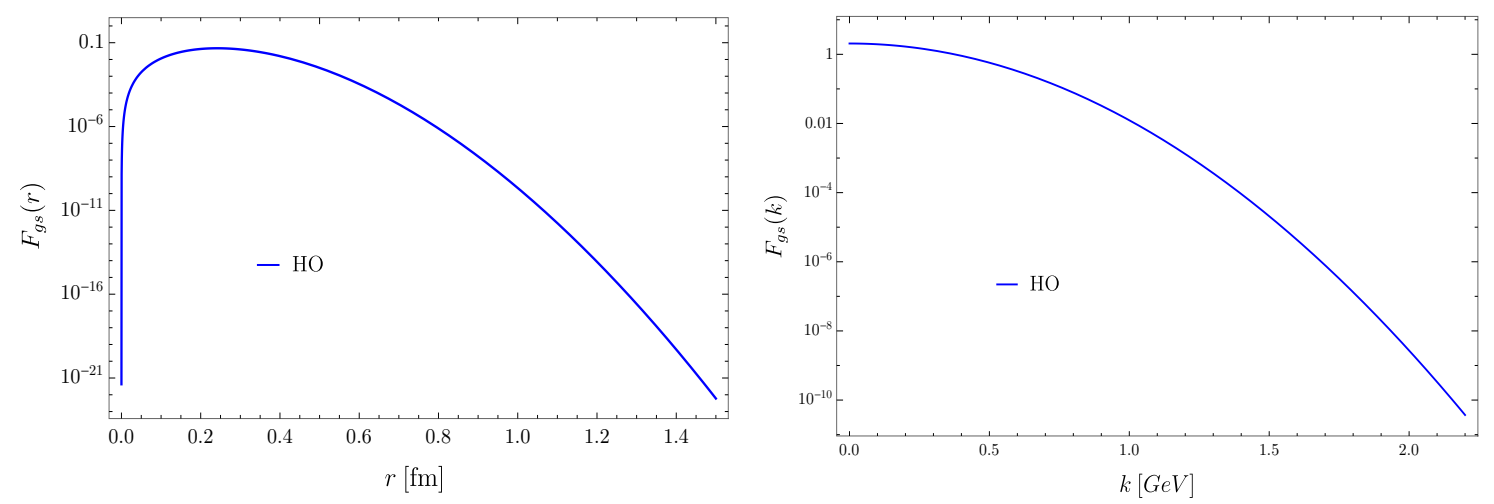

Figure 2: Orbital contribution to the pentaquark photocouplings in the harmonic oscillator quark model calculated with Eq. (14). The left panel shows the result as a function of the pentaquark charge radius $r$ for a fixed value of the photon momentum $k=2.12 \mathrm{GeV}$, and the right panel as a function of the photon momentum for a fixed value of the charge radius $r=1 \mathrm{fm}$.

where $a$ represents the ratio of the heavy and light quark masses $a=m^{\prime} / m$. In the rest frame of the pentaquark, the orbital contribution to the photoproduction of the pentaquark can be derived as

$$
F_{g s}(k)=\left(\frac{\sqrt{5(2 a+3)}}{3}\right)^{\frac{3}{4}}\left(\frac{2 \alpha_{\rho} \beta}{\alpha_{\rho}^{2}+\beta^{2}}\right)^{3} e^{-\frac{5}{12 \alpha_{\rho}^{2}} \sqrt{\frac{2 a+3}{5 a}} k^{2}},
$$

where $\beta$ is fitted to the proton charge radius, $\beta=1 / r_{\mathrm{ch}}(p)$ and $\alpha_{\rho}$ is related to pentaquark charge radius $r$ according to Eq. (13). Since the charge radius of the pentaquark is not known, the results are shown in Fig. 2 as a function of $r$. The photon momentum of $k=2.12 \mathrm{GeV}$ corresponds to a pentaquark with mass $m_{P_{c}}=4.450 \mathrm{GeV}$. The orbital matrix element is found to be very small, with the exception of a pentaquark charge radius around $0.2-0.3 \mathrm{fm}$. The right-hand panel of Fig. 2 shows the dependence on the photon momentum for a fixed value of the pentaquark charge radius of $1 \mathrm{fm}$.

\section{Summary and conclusions}

In this contribution we discussed the photocouplings of ground-state $q q q Q \bar{Q}$ pentaquarks in the harmonic oscillator quark model in which we distinguish between light and heavy quarks. As a consequence of the large photon momentum $(k=2.12 \mathrm{GeV})$, the photocouplings are strongly suppressed by the orbital contribution. A similar result is obtained for excited-state pentaquark configurations [27]. These results may help to explain why the signals obtained by the LHCb collaboration have not been observed in experiments with electromagnetic probes. On the other hand, the fact that the pentaquarks have not been observed experimentally in photoproduction experiments does not exclude that the signals observed by $\mathrm{LHCb}$ correspond to compact pentaquark states.

\section{Acknowledgments}

This work was supported by grant IN101320 from PAPIIT, DGAPA-UNAM. 


\section{References}

[1] R. Aaij et al. (LHCb), Phys. Rev. Lett. 115, 072001 (2015), arXiv:1507.03414 [hep-ex] .

[2] R. Aaij et al. (LHCb), Phys. Rev. Lett. 117, 082002 (2016), arXiv:1604.05708 [hep-ex] .

[3] R. Aaij et al. (LHCb Collaboration), Phys. Rev. Lett. 122, 222001 (2019).

[4] F.-K. Guo, U.-G. Meißner, W. Wang, and Z. Yang, Phys. Rev. D92, 071502 (2015), arXiv:1507.04950 [hep-ph] .

[5] X.-H. Liu, Q. Wang, and Q. Zhao, AIP Conference Proceedings 1735, 060004 (2016), https://aip.scitation.org/doi/pdf/10.1063/1.4949440 .

[6] U.-G. Meißner and J. A. Oller, Phys. Lett. B751, 59 (2015), arXiv:1507.07478 [hep-ph] .

[7] H.-X. Chen, W. Chen, X. Liu, T. G. Steele, and S.-L. Zhu, Phys. Rev. Lett. 115, 172001 (2015), arXiv:1507.03717 [hep-ph] .

[8] L. Roca, J. Nieves, and E. Oset, Phys. Rev. D92, 094003 (2015), arXiv:1507.04249 [hep-ph] .

[9] J. He, Phys. Lett. B753, 547 (2016), arXiv:1507.05200 [hep-ph] .

[10] Q.-F. Lü and Y.-B. Dong, Phys. Rev. D93, 074020 (2016), arXiv:1603.00559 [hep-ph] .

[11] Z. Meziani et al. (Hall A), E12-12-006: Near Threshold Electroproduction of J/4 at $11 \mathrm{GeV}$ (2012).

[12] S. Stepanyan et al. (Hall B), E12-12-001A Near Threshold J/ $\psi$ Photoproduction and Study of LHCb Pentaquarks with CLAS12 (2017).

[13] Z. E. Meziani et al. (Hall C), E12-16-007: A Search for the LHCb Charmed 'Pentaquark' using Photo-Production of J/ $\psi$ at Threshold in Hall C at Jefferson Lab (2016) arXiv:1609.00676 [hep-ex] .

[14] L. Robison (GlueX), in 2017 Fall Meeting of the APS Division of Nuclear Physics. Pittsburgh, Pennsylvania, October 25-28, 2017 (2017).

[15] D. Winney, C. Fanelli, A. Pilloni, A. N. H. Blin, C. Fernández-Ramírez, M. Albaladejo, V. Mathieu, V. I. Mokeev, and A. P. Szczepaniak (Joint Physics Analysis Center), Phys. Rev. D 100, 034019 (2019).

[16] A. Ali et al. (GlueX Collaboration), Phys. Rev. Lett. 123, 072001 (2019).

[17] S. G. Yuan, K. W. Wei, J. He, H. S. Xu, and B. S. Zou, Eur. Phys. J. A48, 61 (2012), arXiv:1201.0807 [nucl-th] .

[18] L. Maiani, A. D. Polosa, and V. Riquer, Phys. Lett. B749, 289 (2015), arXiv:1507.04980 [hep-ph] .

[19] R. F. Lebed, Phys. Lett. B749, 454 (2015), arXiv:1507.05867 [hep-ph] . 
[20] A. Mironov and A. Morozov, JETP Lett. 102, 271 (2015), [Pisma Zh. Eksp. Teor. Fiz.102,no.5,302(2015)], arXiv:1507.04694 [hep-ph] .

[21] E. Santopinto and A. Giachino, Phys. Rev. D96, 014014 (2017), arXiv:1604.03769 [hep-ph] .

[22] E. Ortiz-Pacheco, R. Bijker, and C. Fernández-Ramírez, Journal of Physics G: Nuclear and Particle Physics 46, 065104 (2019).

[23] A. Le Yaouanc, L. Oliver, O. Pene, and J. C. Raynal, Hadron Transitions in the Quark Model (Gordon and Breach, New York, NY, USA, 1988).

[24] L. A. Copley, G. Karl, and E. Obryk, Nucl. Phys. B13, 303 (1969).

[25] F. E. Close, An Introduction to Quarks and Partons (Academic Press, London, UK, 1979).

[26] R. Bijker, F. Iachello, and A. Leviatan, Annals of Physics 284, 89 (2000).

[27] E. Ortiz-Pacheco and R. Bijker, to be published . 